\title{
AC Electrowetting Modulation of Low-Volatile Liquids Probed by XPS: Dipolar vs lonic Screening
}

\author{
Pinar Aydogan Gokturk, ${ }^{\circledR}$ Burak Ulgut, ${ }^{\circledR}$ and Sefik Suzer*(-) \\ Department of Chemistry, Bilkent University, Bilkent, 06800 Ankara, Turkey \\ Supporting Information
}

\begin{abstract}
X-ray photoelectron spectroscopic (XPS) data have been recorded for a low-molecular-weight poly(ethylene glycol) microliter-sized sessile liquid drops sitting on a dielectric covered planar electrode while imposing a $\pm 6 \mathrm{~V}$ square-wave actuation with varying frequencies between $10^{-1}$ and $10^{5} \mathrm{~Hz}$ to tap into the information derivable from (AC) electrowetting. We show that this time-varying XPS spectra reveal two distinct behaviors of the device under investigation, below and above a critical frequency, measured as $\sim 70 \mathrm{~Hz}$ for the liquid poly(ethylene glycol) with a $600 \mathrm{Da}$ molecular weight. Below the critical frequency, the liquid complies faithfully to the applied bias, as determined by the constant shift in the binding energy position of the XPS peaks representative of the liquid throughout its entire surface. The liquid completely screens the applied electrical field and the entire potential drop takes place at the liquid/

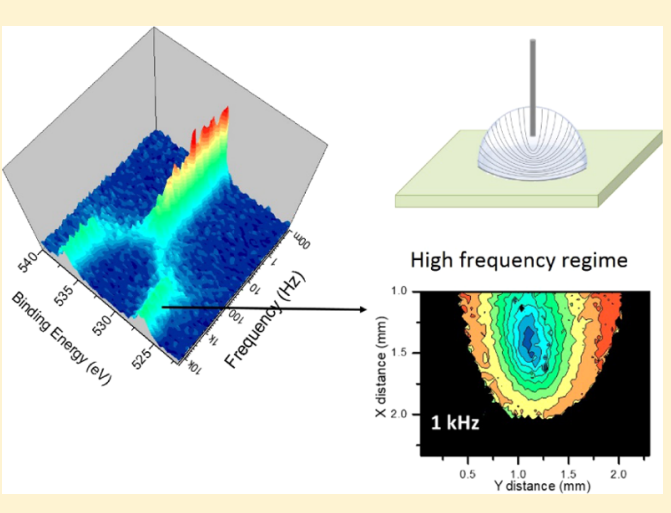
dielectric interface. However, for frequencies above the critical value, the resistive component of the system dominates, resulting in the formation of equipotential surface contours, which are derived from the differences in the positions of the twinned $\mathrm{O} 1 \mathrm{~s}$ peaks under AC application. This critical frequency is independent of the size of the liquid drop, and the amplitude of the excitation, but increases when ionic moieties are introduced. The XP spectra under AC actuation is also faithfully simulated using an equivalent circuit model consisting of only resistors and capacitors and using an electrical circuit simulation software. Moreover, a mimicking device is fabricated and its XP spectra are recorded using the Sn 3d peaks of the solder joints at different points on the circuit to confirm the reliability of the measured and simulated AC behaviors of the liquid. These new findings indicate that in contrast to direct current case, XPS measurements under variable frequency AC actuation reveal (through differences in the frequency response) information related to the chemical makeup of the liquid(s) and brings the laboratorybased XPS as a powerful complimentary arsenal to electrochemical analyses of liquids and their interfaces.
\end{abstract}

\section{INTRODUCTION}

Electrowetting on dielectrics (EWOD) is a process of changing the contact angle of a liquid droplet sitting on a dielectriccovered conducting electrode by application of external electric field(s). Spreading of a liquid drop under an electric field can be observed on both dielectric coated and conductive surfaces as a result of decreasing contact angle by additional electrical force(s). However, the dielectric layer prevents the passage of Faradic current and eliminates the possibility of electrolysis during the process. Hence, larger amplitudes of contact angle changes are achieved. This activation can be realized under both direct current (DC) and alternating current (AC) fields with variable frequency. ${ }^{1-6}$ In fact, it has been reported that the contact-angle hysteresis is smaller in AC and DC, which is why AC is preferred in many applications. ${ }^{7,8}$ One of the major obstacles in both DC and AC actuations is related to the saturation of the contact angle at high voltages, and numerous techniques have been developed to circumvent it. ${ }^{9-11}$ Furthermore, many experimental results have shown that the contact angle saturation occurs at higher effective voltages for the AC when compared to the DC electrowetting., ${ }^{6,12,13}$
Wettability of solid surfaces has great importance for both natural and industrial processes. For this reason, contact angle tunability with application of external bias is crucial and electrowetting offers an excellent option for manipulating small amounts of liquids. Indeed, this technology has been used in various applications such as lab on a chip, ${ }^{14}$ liquid-based lenses, ${ }^{15}$ electronic paper(s), ${ }^{16}$ and electrofluidic displays. ${ }^{17}$ Electrowetting on dielectric behavior is conventionally modeled by an analogy to equivalent electric circuits adopted from the model(s) used for solid-sate devices, one of which is depicted in Figure 1, together with our experimental setup. $^{1,3,18,19}$ In this model, the liquid drop is represented by a parallel resistor and a capacitor, whereas the dielectric layer(s) as another capacitor, and the two electrical double layers formed at the liquid/top and the liquid/bottom electrodes are usually not taken into account. This model is also supported by numerous electrical impedance spectroscopic data. ${ }^{20}$

Received: December 9, 2018

Revised: February 9, 2019

Published: February 15, 2019 


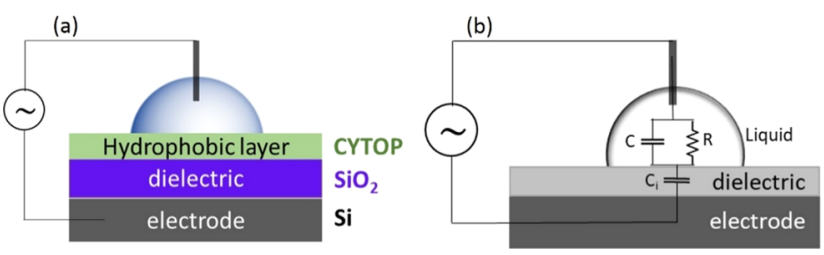

Figure 1. Schematic representation of (a) our experimental setup and (b) equivalent circuit diagram of a droplet on a dielectric-covered electrode.

Using this model for aqueous liquids, researchers have tried to understand the electrical behavior of EWOD. According to the model and at very low frequencies, the droplet behaves like a conductor, hence the entire voltage drop occurs in the dielectric layer and it behaves like a perfect dielectric at very high frequencies. ${ }^{21}$ This behavior is attributed to the presence of the dissolved ions in the liquid, which can follow up the applied bias and screen the electrical field from the interior of the liquid up to a certain frequency. Above this frequency, the voltage increase triggers activation of the displacement currents and formation of potential distribution inside the droplet. ${ }^{1}$

The critical frequency between these two behaviors is determined by the following equation ${ }^{2}$

$$
f_{\mathrm{c}}=\frac{\sigma_{1}}{2 \pi\left(\varepsilon_{1}+\varepsilon_{\mathrm{d}} R / d\right)}
$$

where $\varepsilon_{1}$ and $\varepsilon_{\mathrm{d}}$ are the electrical permittivities for the liquid droplet and the dielectric layer, respectively; $\sigma_{1}$ is the conductivity of the liquid; $R$ is the radius of the droplet; and $d$ is the thickness of the dielectric layer. Beyond the critical frequency, a fraction of the potential that is applied to the wire drops within the droplet and results in the decrease of the effective contact angle change. In the classical electrowetting theory, the liquid is assumed to be a perfect conductor. This property can be achieved by either with the high ionic concentrations or low-frequency applications. On the other hand, it was previously shown that even deionized water can undergo electrowetting at low frequencies, and the critical frequency was reported to be larger for ionic aqueous solutions by Jones and Wang. ${ }^{21}$ In that work, the electric fields created within different types of liquids were also calculated from elementary electrostatics. Using the Maxwell stress tensor, they determined the height of the rise of the liquid to model the contact angle change with varying frequencies. In a later report, Liu et al. had claimed that the performance of EWOD devices does not have a strong dependence on the properties of the liquid but mainly on the properties of the dielectric layer(s). ${ }^{22}$ Therefore, over the last decade, many studies have been reported on improving the chemical and physical makeup of dielectric(s), especially for low-voltage actuation. ${ }^{23}$ However, dielectric failure becomes more pronounced in low-voltage applications, and it was reported that the chemical nature of both ions and liquids utilized can be optimized for the elimination of such failure. ${ }^{24}$ Use of electrochemical impedance spectroscopic measurements, mostly on aqueous solutions, have also been reported for better understanding and control of the material properties for such low-voltage actuations. ${ }^{25}$

Recently, we have reported on an investigation of the DC behavior of a nonaqueous and nonionic liquid, a lowmolecular-weight ( $\sim 600 \mathrm{Da})$ poly(ethylene glycol) (PEG), within the X-ray photoelectron spectrometer (XPS) chamber, in the traditional sessile drop electrowetting on dielectric geometry, for the first time in literature. ${ }^{26}$ The low-molecularweight liquid PEG is suitable for XPS analysis, since it has negligible vapor pressure. PEG has long been and is still being investigated as a suitable polymer electrolyte for battery applications. ${ }^{27}$ It has also been utilized in several green chemistry applications, such as personal care products and food industry. ${ }^{28}$ It is also biodegradable and has the potential for use in droplet-based microfluidics for biological applications. ${ }^{29,30}$ Although PEG itself has not been directly used as a microfluidic liquid medium, it is an important ingredient of the aqueous two-phase systems in which poly(ethylene glycol)-rich phase and dextrene-rich phase constitute the two immiscible liquids. ${ }^{31,32}$ Moreover, both DC and AC electrical fields are used to enhance the flow properties of the fluidic phases. ${ }^{33-35}$ Use of PEG for XPS analysis brings an added simplicity, since both carbon and oxygen atoms are in single chemical states. Moreover, because of the absence of ions, except for impurities, it is expected that the imposed electric field interacts predominantly with the connected dipoles of the liquid structure, the so-called "segmental-motion". ${ }^{36}$

In our previous work, and using the $\mathrm{C} 1 \mathrm{~s}$ intensity maps, we had demonstrated that the PEG droplet spreads on the dielectric-coated substrate with the application of an electrical bias and retracts upon its removal. ${ }^{26}$ However, more importantly, we were able to trace the electrical potential developments on and around the droplet under DC excitation using the shifts in the binding energy positions of XPS peaks of the liquid and the substrate separately. We established that whereas no potential drop is observable at the top wire electrode/liquid interface, the entire potential drop develops across the liquid/substrate interface faster than our probe time, up to large biases leading to the dielectric breakdown around $120 \mathrm{~V}$. It was also demonstrated that no band bending nor broadening in the $\mathrm{C} 1 \mathrm{~s}$ peaks was observable even under electrical fields as large as $10^{7} \mathrm{~V} / \mathrm{m}$, within the liquid/solid dielectric interface. However, although different liquids such as PEG (either pristine or with dissolved ions) and ionic liquids were investigated (but not all reported), no chemical information about the liquid(s) under investigations could be extracted from the DC measurements.

In the present study, we go one step further and report AC electrowetting of the same pure nonaqueous PEG liquid, as well as have an inorganic salt $\left(\mathrm{MgCl}_{2}\right)$ dissolved in it. When a DC bias is introduced, only a corresponding shift in the measured binding energy of a given XP peak is observed, ${ }^{26}$ but an $\mathrm{AC}$ modulation, using square waves (SQW), twins all peaks at correspondingly lower and higher binding energy positions, and pertinent electrical information is extractable from the difference of these positions of the twinned peaks, as was demonstrated in our previous work. ${ }^{37,38}$ Similar to the previous DC work, we analyze, using XPS, now under a square-wave AC electrical signal with varying frequency, and record electrical potential developments on and around the sessile droplet placed on a $\sim 300 \mathrm{~nm} \mathrm{SiO}{ }_{2}$ dielectric substrate covered with a thin $(\sim 50 \mathrm{~nm})$ and hydrophobic polymer layer. We also utilize simple AC electrical and impedance measurements to provide an input for modeling the measured XPS data using a simulation software. Although large number of experimental and theoretical studies have been devoted to predict the form and frequency dependence, this work is the first to directly measure and model the potential developments on and around liquid droplets in a chemically resolved and noninvasive 
Substrate Biased

(a)
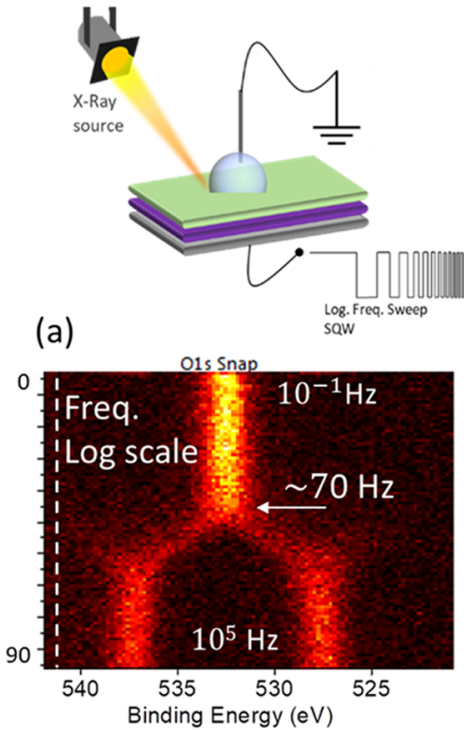

Drop Biased

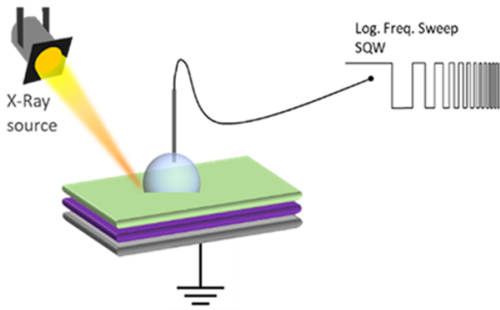

(b)

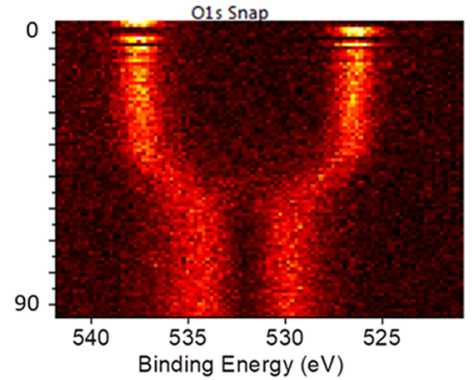

Figure 2. Schematic representation of the AC actuation by imposing the bias from (a) the bottom or (b) the top electrodes, together with the recorded $\mathrm{O} 1 \mathrm{~s}$ spectra, while sweeping the frequency.

fashion. As will be shown below, contrary to the DC case, variable frequency $\mathrm{AC}$ measurements reveal critical and pertinent information related to chemical composition of the liquid(s) under investigation.

\section{EXPERIMENTAL DETAILS}

$\mathrm{MgCl}_{2}$ and PEG were purchased from Sigma-Aldrich and Merck, respectively. Si substrates with $\sim 300 \mathrm{~nm}$ oxide layer are dip-coated with an amorphous fluoropolymer layer and used as the bottom electrode. Approximately, a $3 \mu \mathrm{L}$ PEG drop is placed on top of this insulated electrode using a micropipette. A copper wire is immersed into the droplet via which the bias is introduced between the liquid and the insulated $\mathrm{Si}$ electrode, as shown in Figure 1a. The function generator (Stanford Research Systems DS340) is connected to the Si substrate, whereas the wire, hence the liquid droplet, is ground or vice versa. All experiments are conducted in the ultra-high vacuum chamber of a Thermo Fisher K-Alpha X-ray photoelectron spectrometer, which is also used to collect the XPS data for all measurements. An AC signal in the form of $\mp 6 \mathrm{~V}$ square waves (SQW) is generated and applied either at a fixed frequency or as a logarithmic frequency sweep from $100 \mathrm{mHz}$ to $100 \mathrm{kHz}$. The SQW modulation, rather than conventional sine wave, is adopted, since it introduces the least amount of distortion to the line shapes of the XPS peaks (Figure S2a). Details of the XP spectra in different datagathering modes and their graphical representations are given in the Supporting Information (SI) section. Note that the most important information harvested in this study, which is the critical frequency, turns out to be insensitive to the form of excitation, i.e., the sine or the square wave, as shown in Figure S2b. An equivalent circuit model is also generated using the LT-Spice simulation program, ${ }^{39}$ whose output is convoluted with the unbiased $\mathrm{O} 1 \mathrm{~s}$ peak to generate and compare with the recorded biased XPS data. In addition and for mimicking the liquid behavior, a parallel device is fabricated using solid-state resistors and capacitors, and analyzed by XPS using the same strategy. This time, the $\mathrm{Sn} 3 \mathrm{~d}$ peaks are recorded at different soldier joints along the equivalent circuit. The XPS response of this device is compared with that of the liquid drop.

\section{RESULTS AND DISCUSSION}

AC Actuation of Pristine PEG. A schematic representation of the device in the experimental arrangement is depicted in Figure 2, together with a collection of XP spectra of PEG.
We apply a time-varying SQW electrical signal with a logarithmic frequency sweep, starting from $100 \mathrm{mHz}$ and reaching $100 \mathrm{kHz}$ with an amplitude of $6 \mathrm{~V}$, either from the bottom of the planar electrode (Figure $2 \mathrm{a}$ ) or the top of the liquid drop (Figure $2 \mathrm{~b}$ ). We then follow the surface potentials by recording the $\mathrm{O} 1 \mathrm{~s}$ signal at various points on the droplet surface. When actuated from the bottom electrode, the PEG, a dielectric liquid, adopts a ground potential of the inserted wire electrode at very low frequencies up to $\sim 70 \mathrm{~Hz}$, and only one $\mathrm{O} 1 \mathrm{~s}$ peak is measurable. After $70 \mathrm{~Hz}$, two $\mathrm{O}$ 1s peaks are measured, reflecting the positive and the negative components of the SQW potential bias. The difference in the binding energy positions of these two peaks increases continuously and reaches a steady state value at $\sim 800 \mathrm{~Hz}$, which is maintained at higher frequencies.

The continuous nature of the transition from complete screening to resistive behavior as a function of the frequency is illustrated in Figure 2a, when the bias is applied from the bottom electrode. In the reverse geometry, where the bias is applied through the droplet, whereas the bottom electrode is grounded, the frequency dependence exhibits an inverse and complementary behavior, as seen in Figure $2 b$. Accordingly, we can define the frequencies up to $70 \mathrm{~Hz}$ and above $800 \mathrm{~Hz}$ regions as the low- and high-frequency regimes, respectively. The measured nonvarying onset of $70 \mathrm{~Hz}$ on the entire surface of the droplet can be associated with the critical frequency for the pristine PEG having $\sim 600 \mathrm{Da}$ molecular weight. The corresponding value for the deionized water has been reported and also predicted by numerous scalculations to be in the order of a few $\mathrm{kHz}$ and increases further by the incorporation of ionic components. ${ }^{21}$ In an attempt to actuate the aqueous solution at low voltages, $\mathrm{Li}$ et $\mathrm{al}^{25}$ reported a strong dependence of the measured critical frequency on the amplitude of the applied AC field(s), contrary to our measurements showing that the critical frequency $(70 \mathrm{~Hz})$ and the extent of its variation (up to $800 \mathrm{~Hz}$ ) are constant for SQW amplitudes in the range $2-10 \mathrm{~V}$, as depicted in Figure S3a of the SI. 

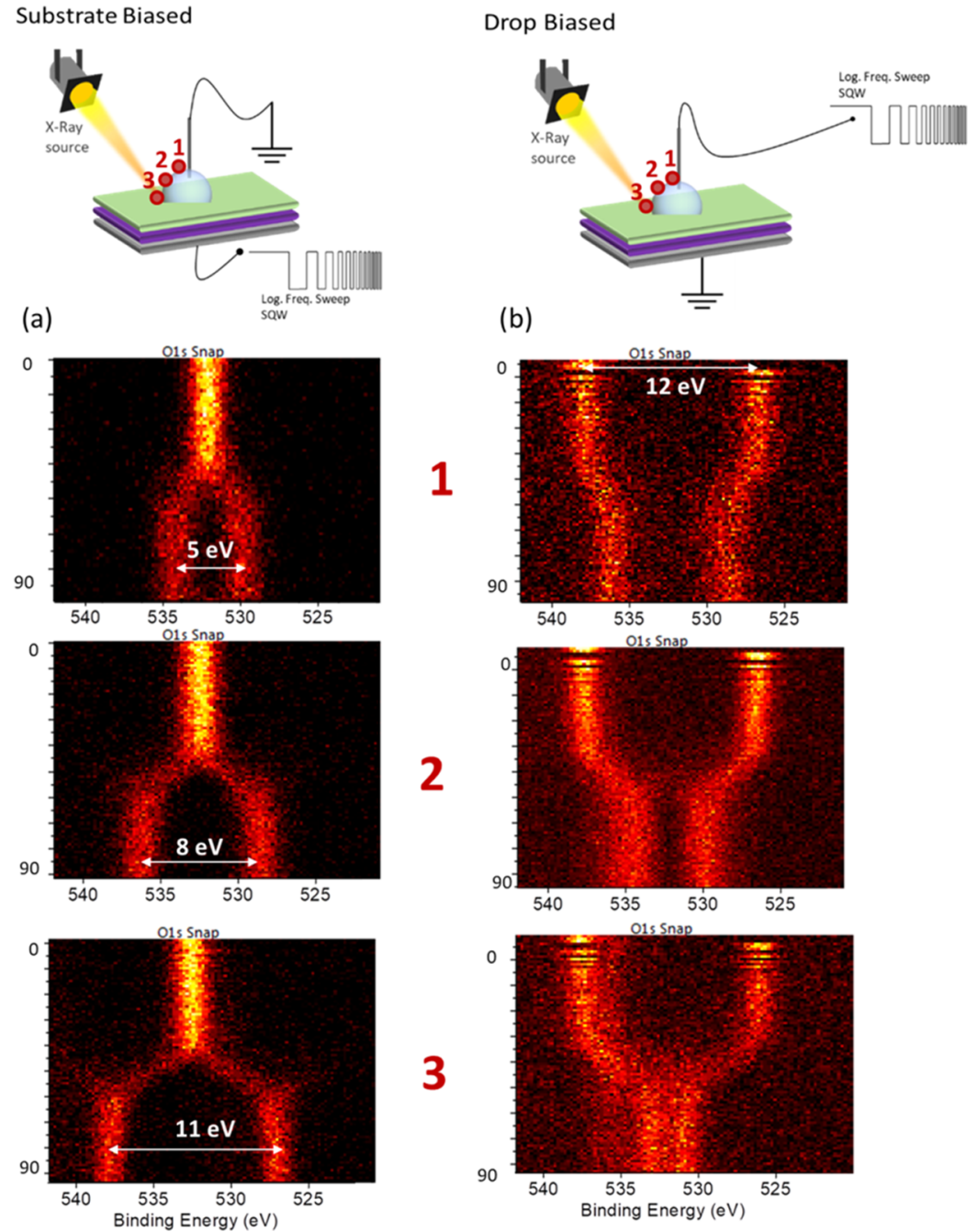

Figure 3. $\mathrm{O}$ 1s spectra recorded under continuous $\mathrm{AC}$ actuation at three different positions on the drop while imposing the bias from (a) the top or (b) the bottom electrodes.

The measured steady-state potential at high frequencies, on the other hand, is a fraction of the bias and its magnitude differs significantly along the droplet surface, as depicted in Figure 3. We, therefore, advocate that the lower critical frequency, as probed by XPS under the SQW modulation, is an intrinsic property of the liquid and is the same for all points analyzed on the entire drop; i.e., it does not change with position. However, steady-state potential at higher frequencies depends strongly on the position and adapts to a fraction of the applied potential as a function of the distance of the analyzed point from the wire electrode, as depicted in Figure 3a,b, which shows iterative $\mathrm{O} 1 \mathrm{~s}$ signals recorded at three different positions on the drop.

As can be gathered from the figure, whereas the lower critical frequency stays constant, the amplitude of the potential measured varies with the distance from the electrodes at higher frequencies. Thus, at a point close to the liquid/dielectric interface, negative and positive components of the $\mathrm{O} 1 \mathrm{~s}$ peak merges (near zero IR drop), as opposed to the case of lowerfrequency regime where no IR drop is measurable (full $12 \mathrm{~V}$ ).

To extend these measurements to the entire liquid surface, we have recorded the $\mathrm{O} 1 \mathrm{~s}$ signal in the areal mapping mode and at only two frequencies representative of the lower- and the higher-frequency regimes, since collecting continuous frequency data are not feasible. We then fitted the XP spectrum at each point to two $\mathrm{O} 1 \mathrm{~s}$ peaks, corresponding to the negative and the positive components and took the binding energy difference between them. The binding energy differences of two components are displayed in Figure 4 as areal maps for the lower $(50 \mathrm{~Hz})$ and higher $(1 \mathrm{kHz})$ frequencies separately. Details of the data handling are given in the SI section, as Figure S3.

As is evident at $50 \mathrm{~Hz}$, the liquid completely screens the applied electrical field and the entire potential drop takes place at the liquid/dielectric interface. As a result, only one $\mathrm{O} 1 \mathrm{~s}$ peak is observed. However, at $1 \mathrm{kHz}$, the liquid can no longer screen the field, and the drop behaves like a resistive medium, resulting in splitting of the $\mathrm{O} 1 \mathrm{~s}$ peaks. The magnitude of the splitting is near zero at the tip of the top electrode and increases to up to $12 \mathrm{eV}$ at the liquid/dielectric interface. This behavior is evidenced by the equipotential surface contours in Figure $4 \mathrm{~b}$.

Simulation of XP Spectra. In light of the above findings and also additional measurements we carried out using a mimicking device to be described below, a simple representation of the system using an equivalent circuit can be realized by assigning a series of resistors and one parallel capacitor for the drop and a very large leakage resistor and a capacitor for 


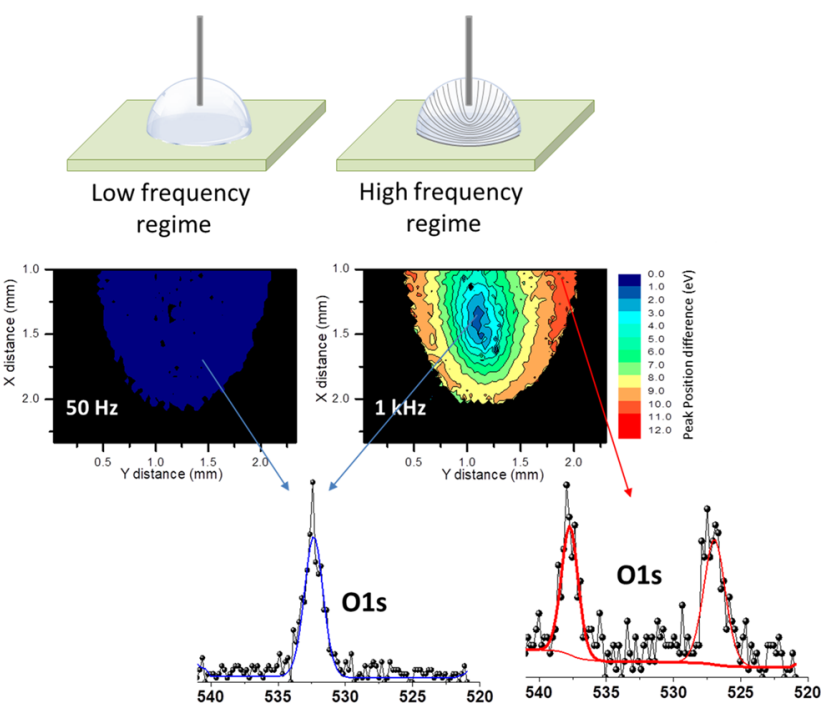

Figure 4. Areal maps of the binding energy difference of positive and negative components of $\mathrm{O} 1 \mathrm{~s}$ under the applied $6 \mathrm{~V}$ square wave bias from the bottom electrode with (a) $50 \mathrm{~Hz}$ and (b) $1 \mathrm{kHz}$ frequencies together with their schematic representations.

dielectric as shown in Figure 5. Note that although an infinite number of series resistors should have been used to represent the liquid drop, only four are adopted for practical purposes. In addition, we, like many studies using the equivalent electrical circuits for EWOD, do not consider the electrical double layers on the interfaces, since the double-layer thicknesses are much smaller than the thickness of the dielectric. As a result, the dielectric capacitance dominates, which has been proven by many other groups that the error stemming from ignoring the double layer, for a planar dielectric/liquid interface, is in the order of $0.01 \% .^{20-23}$ Accordingly, we can estimate the capacitance of the dielectric layer, hence that of the system, to be $\sim 270 \mathrm{pF}$ using the geometry of the drop and the dielectric parameters of the media.

Using this value and the measured lower frequency limit of $70 \mathrm{~Hz}$, the simplified equivalent circuit consisting of a series $R_{\text {liquid }}$ and $C_{\text {dielectric }}$ the total effective AC resistance of the drop can now be estimated as $\sim 8 \mathrm{M} \Omega$. Details of the calculations are given in the SI. In the next step, we used the output waveforms generated with the simulation software, LT-Spice, ${ }^{39}$ from the equivalent circuit using these values to generate the XPS data at two different points along the drop and at several relevant frequencies, which are shown in Figure 5a,b. In the same figure, the corresponding measured spectra are also reproduced in Figure 5c,d, which are given in Figure 3. Resemblance of the simulated data to those of the measured ones is simply remarkable.

One significant outcome of the XPS measurements and their successful simulations is that, although the model has been developed mainly for aqueous and conducting liquids, it can also be applied to dipolar but reasonably nonconducting liquid(s) (for the PEG drops used in this work, the estimated $\mathrm{DC}$ resistance is larger than $50 \mathrm{M} \Omega$ ).

Mimicking Liquid Actuation. To further verify the simulation results, we have carried out AC XPS measurements on a mimicking device made out of only resistors and capacitors connected together by soldering. The Sn $3 \mathrm{~d}$ peak at the soldering connections are used to record the data at four different points, representative of the different points along the liquid drop, as given in Figure 3. The data are displayed in Figure 6. Examining the spectra displayed in these two figures leads us to confidently postulate that the device faithfully mimics the liquid drop.

AC Actuation of $\mathrm{MgCl}_{2}$ Dissolved PEG. As expressed in eq 1 , the relevant critical frequency in electrowetting stems out

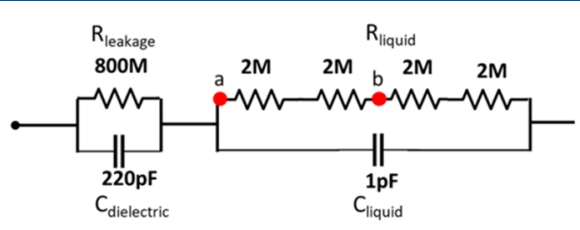

(a)

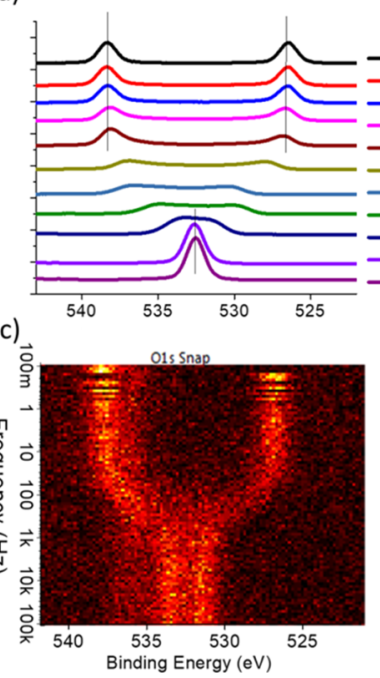

(b)

(d)
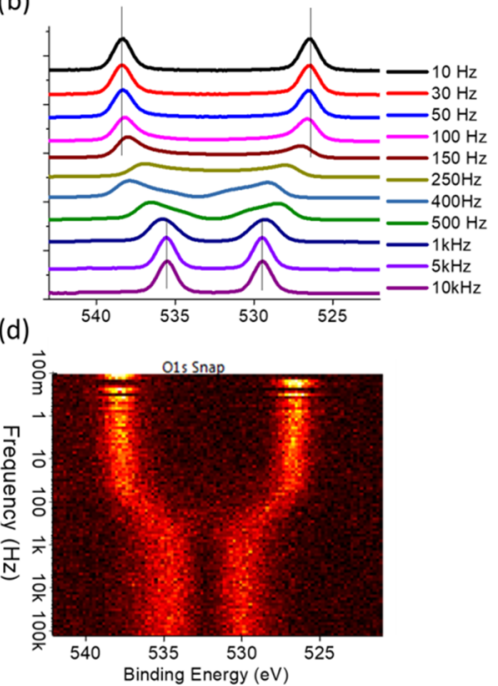

Figure 5. (a, b) Simulated O 1s spectra at two different positions on the equivalent circuit used, whereas (c) and (d) are the recorded ones on the liquid surface at two different points. 

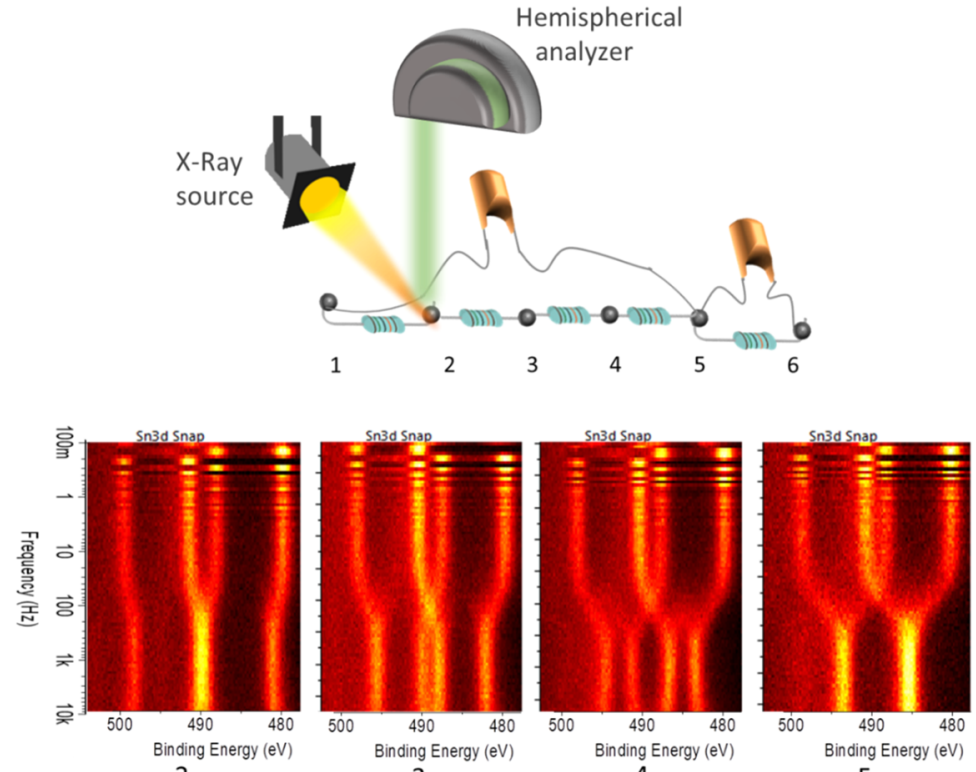

Biased from left end
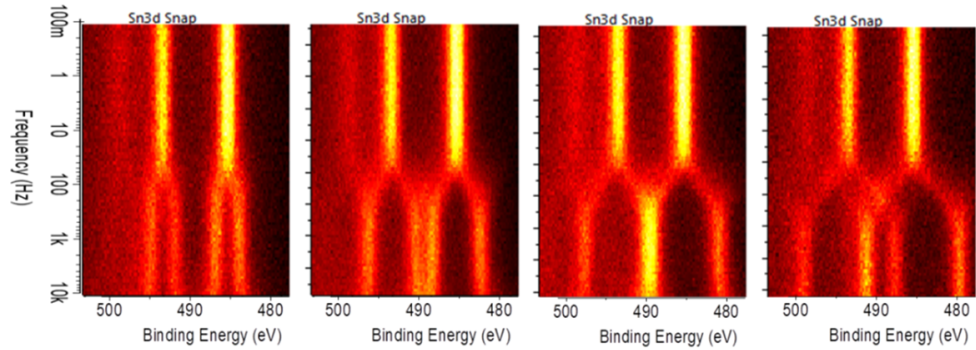

Biased from

right end

Figure 6. Sn 3d spectra recorded at four different positions on the mimicking device. Top row corresponds to actuation from the wire electrode, whereas bottom row correspondence to actuation from the planar Si electrode.

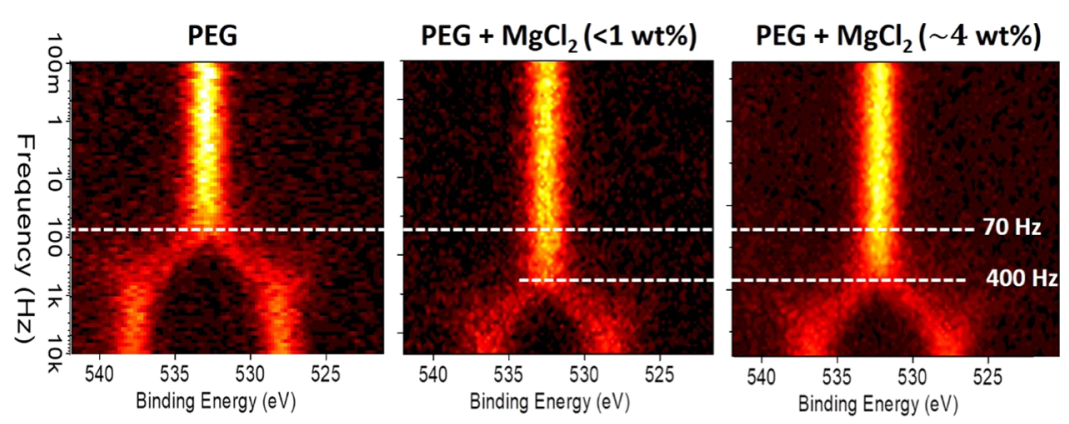

Figure 7. $\mathrm{O}$ 1s spectra recorded under continuous $\mathrm{AC}$ actuation $\left(10^{-1}-10^{4} \mathrm{~Hz}\right)$ for three different liquid drops.

as an intrinsic property of the liquid and is expected to change with the conductivity of the liquid, as was observed for the aqueous ionic solutions. ${ }^{21}$ To confirm this prediction, we have carried out the same type of AC measurements on two additional PEG liquid samples having different concentrations of dissolved $\mathrm{MgCl}_{2}$, which are reproduced in Figure 7.

As predicted, addition of ionic moieties into the PEG media shifts the critical frequency from 70 to $400 \mathrm{~Hz}$, with surprisingly no measurable dependence on the concentration of ions. Similar findings were earlier reported by Di Noto and co-workers, who investigated similar systems using electrochemical impedance spectroscopy for elucidating the mechanism(s) of their electrical conductivity. ${ }^{40-42}$ They suggested that the conductivity of PEG400 $\left(\mathrm{MgCl}_{2}\right)_{x}$ complexes is ionic and takes place via two distinct phenomena: (i) hoping of cationic species $\mathrm{Mg}^{2+}$ and $[\mathrm{MgCl}]^{+}$between the sites along the polyetheral chains (intra-CH hoping) and (ii) migration of ions between different PEG chains (inter- $\mathrm{CH}$ hopping). Their data also suggested that a strong anion-cation interaction was evidenced at high salt concentrations. As a result, equivalent molar ionic conductivity decreases sharply with the increase in salt concentration. These findings are in total agreement with our data that incorporation of small amounts of ions increases the conductivity of the liquid, but further increase in their concentrations does not contribute significantly to the overall conductivity,

We can rationalize our results as the viscous liquid, with its low-molecular-weight polymeric nature has limited and very sluggish and connected dipoles (segmental motion), ${ }^{36}$ which can only screen the applied electrical field at low frequencies. Once, the pulses become faster, the current starts to flow through the liquid. Addition of ions, with higher but again with 
limited mobility in the viscous liquid, introduces ionic screening in addition to the dipolar one, which extends the frequency range of screening, but by only a factor of five.

\section{CONCLUSIONS}

Overall, the outcome of these findings using a new experimental strategy to study $\mathrm{AC}$ electrowetting phenomenon shows several important information related to the electrical potential developments on the surface of the liquid sessile drops. First, at low frequencies, the drop adopts the potential of the bias, leading to uniform and constant potential distribution on its entire surface, which is directly demonstrated for the first time experimentally using XPS in a chemical specific fashion. On the other hand, at higher frequencies, the applied voltage to the wire drops across the droplet and a potential distribution is measured as equipotential contours. Additionally, our measurements suggest that the critical frequency, separating the screening and the resistive behaviors of the liquid drop, is an intrinsic property of the liquid that increases by introducing ions. However, further increase in ion concentration seems not to lead to further increase in the frequency for the viscous liquid investigated. Our findings are also supported by an equivalent circuit model to electrically describe the system under investigation, which is further used to generate simulated XPS spectral data to be compared with both computationally and experimentally using a mimicking device. Our new experimental strategy is simply to implement to the existing lab-based XPS instruments; therefore, we expect it to be adopted by many researchers in the field and used for interrogation of numerous electrochemical obstacles related to the emerging applications of liquids and their interfaces, especially in battery- and energy-related fields. One natural extension of the technique is to employ a combination of DC together with AC actuation, expected to be especially useful for improving the electrowetting performance and geometrical constraints of the devices used. ${ }^{39,40}$

\section{ASSOCIATED CONTENT}

\section{S Supporting Information}

The Supporting Information is available free of charge on the ACS Publications website at DOI: 10.1021/acs.langmuir.8b04099.

Data-gathering modes used, additional data for different AC waveforms and amplitudes and estimation of the parameters for equivalent circuit that mimics the EWOD behavior of $\mathrm{PEG}$ on $\mathrm{CYTOP} / \mathrm{SiO}_{2}$; peak fitting of areal maps and constructing two-dimensional contour plots of potential distribution (PDF)

\section{AUTHOR INFORMATION}

\section{Corresponding Author}

*E-mail: suzer@fen.bilkent.edu.tr.

\section{ORCID}

Pinar Aydogan Gokturk: 0000-0001-8269-8884

Burak Ulgut: 0000-0002-4402-0033

Sefik Suzer: 0000-0002-5866-2600

\section{Author Contributions}

The manuscript was written through contributions of all authors. All authors have given approval to the final version of the manuscript.

\section{Notes}

The authors declare no competing financial interest.

\section{ACKNOWLEDGMENTS}

This work was partially supported by the Scientific and Technological Research Council of Turkey (TUBITAK) grant no. $215 Z 534$.

\section{REFERENCES}

(1) Mugele, F.; Baret, J.-C. Electrowetting: from basics to applications. J. Phys.: Condens. Matter 2005, 17, R705.

(2) Hong, J. S.; Ko, S. H.; Kang, K. H.; Kang, I. S. A numerical investigation on AC electrowetting of a droplet. Microfluid. Nanofluid. 2008, 5, 263-271.

(3) Jones, T. B.; Fowler, J. D.; Chang, Y. S.; Kim, C.-J. FrequencyBased Relationship of Electrowetting and Dielectrophoretic Liquid Microactuation. Langmuir 2003, 19, 7646-7651.

(4) Ko, S. H.; Lee, H.; Kang, K. H. Hydrodynamic Flows in Electrowetting. Langmuir 2008, 24, 1094-1101.

(5) Lee, H.; Yun, S.; Ko, S. H.; Kang, K. H. An electrohydrodynamic flow in ac electrowetting. Biomicrofluidics 2009, 3, No. 044113.

(6) Nanayakkara, Y. S.; Perera, S.; Bindiganavale, S.; Wanigasekara, E.; Moon, H.; Armstrong, D. W. The Effect of AC Frequency on the Electrowetting Behavior of Ionic Liquids. Anal. Chem. 2010, 82, $3146-3154$

(7) Bhushan, B.; Pan, Y. Role of Electric Field on Surface Wetting of Polystyrene Surface. Langmuir 2011, 27, 9425-9429.

(8) Li, F.; Mugele, F. How to make sticky surfaces slippery: Contact angle hysteresis in electrowetting with alternating voltage. Appl. Phys. Lett. 2008, 92, No. 244108.

(9) Quinn, A.; Sedev, R.; Ralston, J. Contact Angle Saturation in Electrowetting. J. Phys. Chem. B 2005, 109, 6268-6275.

(10) Yamamoto, T.; Masao, D.; David, A. Contact angle saturation in electrowetting: Injection of ions into the surrounding media. Europhys. Lett. 2015, 112, No. 56001.

(11) Papathanasiou, A. G.; Papaioannou, A. T.; Boudouvis, A. G. Illuminating the connection between contact angle saturation and dielectric breakdown in electrowetting through leakage current measurements. J. Appl. Phys. 2008, 103, No. 034901.

(12) Paneru, M.; Priest, C.; Sedev, R.; Ralston, J. Static and Dynamic Electrowetting of an Ionic Liquid in a Solid/Liquid/Liquid System. J. Am. Chem. Soc. 2010, 132, 8301-8308.

(13) Paneru, M.; Priest, C.; Sedev, R.; Ralston, J. Electrowetting of Aqueous Solutions of Ionic Liquid in Solid-Liquid-Liquid Systems. J. Phys. Chem. C 2010, 114, 8383-8388.

(14) Pollack, M. G.; Fair, R. B.; Shenderov, A. D. Electrowettingbased actuation of liquid droplets for microfluidic applications. Appl. Phys. Lett. 2000, 77, 1725-1726.

(15) Kuiper, S.; Hendriks, B. H. W. Variable-focus liquid lens for miniature cameras. Appl. Phys. Lett. 2004, 85, 1128-1130.

(16) Hayes, R. A.; Feenstra, B. J. Video-speed electronic paper based on electrowetting. Nature 2003, 425, 383.

(17) Heikenfeld, J.; Zhou, K.; Kreit, E.; Raj, B.; Yang, S.; Sun, B.; Milarcik, A.; Clapp, L.; Schwartz, R. Electrofluidic displays using Young-Laplace transposition of briliant pigment dispersions. Nat. Photonics 2009, 3, 292-296.

(18) Chen, L.; Bonaccurso, E. Electrowetting - From statics to dynamics. Adv. Colloid Interface Sci. 2014, 210, 2-12.

(19) Nelson, W. C.; Kim, C.-J. C. Droplet Actuation by Electrowetting-on-Dielectric (EWOD): A Review. J. Adhes. Sci. Technol. 2012, 26, 1747-1771.

(20) Hu, X.; Mibus, M.; Knospe, C. R.; Zangari, G.; Reed, M. L. Impedance spectroscopy and electrical modeling of electrowetting on dielectric devices. J. Micromech. Microeng. 2015, 25, No. 045020.

(21) Jones, T. B.; Wang, K. L.; Yao, D. J. Frequency-Dependent Electromechanics of Aqueous Liquids: Electrowetting and Dielectrophoresis. Langmuir 2004, 20, 2813-2818. 
(22) Liu, H.; Dharmatilleke, S.; Maurya, D. K.; Tay, A. A. O. Dielectric materials for electrowetting-on-dielectric actuation. Microsyst. Technol. 2010, 16, 449-460.

(23) Narasimhan, V.; Park, S.-Y. An Ion Gel as a Low-Cost, SpinCoatable, High-Capcitance Dielectric for Electrowetting-on-Dielectric (EWOD). Langmuir 2015, 31, 8512-8518.

(24) Raj, B.; Dhindsa, M.; Smith, N. R.; Laughlin, R.; Heikenfeld, J. Langmuir 2009, 25, 12387-12392.

(25) Li, Y.-J.; Cahill, B. P. Frequency Dependence of Low-Voltage Electrowetting Investigated by Impedance Spectroscopy. Langmuir 2017, 33, 13139-13147.

(26) Aydogan Gokturk, P.; Ulgut, B.; Suzer, S. DC Electrowetting of Nonaqueous Liquid Revisited by XPS. Langmuir 2018, 34, 73017308.

(27) Wright, P. V. Polymer electrolytes-the early days. Electrochim. Acta 1998, 43, 1137-1143.

(28) Chen, J.; Spear, S. K.; Huddleston, J. G.; Rogers, R. D. Polyethylene glycol and solutions of polyethylene glycol as green reaction media. Green Chem. 2005, 7, 64-82.

(29) Knop, K.; Hoogenboom, R.; Fischer, D.; Schubert, U. S. Poly(ethylene glycol) in Drug Delivery: Pros and Cons as Well as Potential Alternatives. Angew. Chem., Int. Ed. 2010, 49, 6288-6308.

(30) Aschenbrenner, O.; Supasitmongkol, S.; Taylor, M.; Styring, P. Measurement of vapour pressures of ionic liquids and other low vapour pressure solvents. Green Chem. 2009, 11, 1217-1221.

(31) Ziemecka, I.; van Steijn, V.; Koper, G. J. M.; Rosso, M.; Brizard, A. M.; van Esch, J. H.; Kreutzer, M. T. Monodisperse hydrogel microspheres by forced droplet formation in aqueous two-phase systems. Lab Chip 2011, 11, 620-624.

(32) Geschiere, S. D.; Ziemecka, I.; van Steijn, V.; Koper, G. J. M.; van Esch, J. H.; Kreutzer, M. T. Slow growth of the Rayleigh-Plateau instability in aqueous two phase systems. Biomicrofluidics 2012, 6, No. 022007.

(33) Link, D. R.; Grasland-Mongrain, E.; Duri, A.; Sarrazin, F.; Cheng, Z.; Cristobal, G.; Marquez, M.; Weitz, D. A. Electric Control of Droplets in Microfluidic Devices. Angew. Chem., Int. Ed. 2006, 45, $2556-2560$.

(34) Gu, H.; Malloggi, F.; Vanapalli, S. A.; Mugele, F. Electrowetting-enhanced microfluidic device for drop generation. Appl. Phys. Lett. 2008, 93, No. 183507.

(35) Tan, S. H.; Semin, B.; Baret, J.-C. Microfluidic flow-focusing in ac electric fields. Lab Chip 2014, 14, 1099-1106.

(36) Ratner, M. A.; Shriver, D. F. Ion transport in solvent-free polymers. Chem. Rev. 1988, 88, 109-124.

(37) Sezen, H.; Suzer, S. XPS for chemical- and charge-sensitive analyses. Thin Solid Films 2013, 534, 1-11.

(38) Sezen, H.; Ozbay, E.; Suzer, S. XPS for probing the dynamics of surface voltage and photovoltage in GaN. Appl. Surf. Sci. 2014, 323, $25-30$.

(39) https://www.analog.com/en/design-center/design-tools-andcalculators/LTspice-simulator.html?doc=LTspice.pdf.

(40) Di Noto, V.; Longo, D.; Münchow, V. Ion-Oligomer Interactions in Poly(ethylene glycol $) 400 /(\mathrm{LiCl}) \times$ Electrolyte Complexes. J. Phys. Chem. B 1999, 103, 2636-2646.

(41) Di Noto, V. Electrical Spectroscopy Studies of Lithium and Magnesium Polymer Electrolytes Based on PEG400. J. Phys. Chem. B 2002, 106, 11139-11154.

(42) Di Noto, V.; Vittadello, M. Mechanism of ionic conductivity in poly(ethylene glycol 400$) /(\mathrm{MgCl} 2) \mathrm{x}$ polymer electrolytes: studies based on electrical spectroscopy. Solid State Ionics 2002, 147, 309316. 